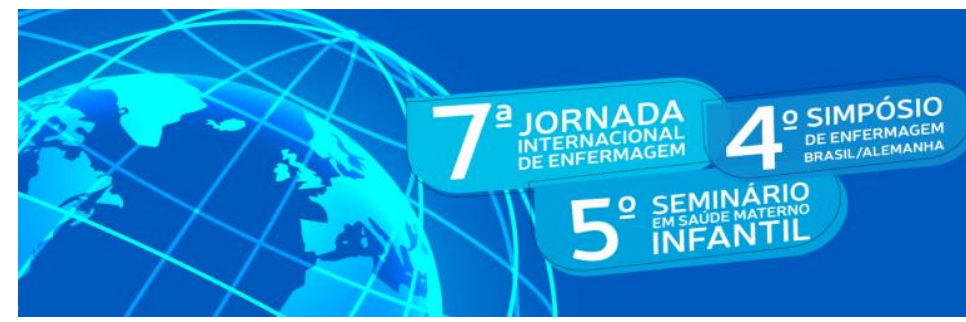

PRÁTICA AVANÇADA EM SAÚDE:

desafios e projeçöes interprofissionais.

05 a 07.05 de 2021

ISSN: 2318-0234

DOI: http://doi.org/10.48195/jie2021-143

\title{
SALAS DE APOIO À AMAMENTAÇÃO E PROLONGAMENTO DO ALEITAMENTO MATERNO POR MULHERES TRABALHADORAS
}

\author{
Gabriele Hatwig knob'; Cristina Saling Kruel²; Evangelia Kotzias Atherino dos Santos ${ }^{3}$; \\ Franceliane Jobim Benedetti ${ }^{4}$; Patrícia Pasquali Dotto ${ }^{5}$
}

\begin{abstract}
RESUMO
No momento atual, é crescente a promoção e o incentivo à implantação de salas de apoio à amamentação nos locais de trabalho. Esse tema vem sendo pesquisado, tanto em âmbito nacional como internacional, dada a importância de proporcionar e apoiar a manutenção do aleitamento materno, seguindo as recomendações da Organização Mundial da Saúde. Isso representa também, um grande desafio, principalmente após o retorno das mulheres ao trabalho, sendo essa a maior barreira para a manutenção da amamentação por parte das mães trabalhadoras. Objetivo científico: Investigar as evidências científicas contempladas na literatura nacional e internacional acerca da importância das salas de amamentação já existentes nas instituições para a manutenção do aleitamento materno por mulheres trabalhadoras. Metodologia: Este estudo é uma revisão narrativa da literatura referente ao aleitamento materno por mulheres trabalhadoras, no local de trabalho e às salas de apoio à amamentação nesses locais. Foram realizadas buscas nas bases de dados LILACS, Medline, Pubmed e em ferramentas como o Google Acadêmico. Resultados: De acordo com a literatura disponível, ao invés de as mães deixarem o mercado de trabalho, deixam os bebês na escola e usam substitutos para o leite materno ou interrompem a amamentação. Dessa forma, as mães geralmente recomendam o acesso a uma sala deste tipo, confortável e equipada, e a creches como fatores de apoio, para que possam voltar ao trabalho e continuarem amamentando seus filhos.
\end{abstract}

Palavras-chave: Aleitamento materno; Local de trabalho; Mulheres trabalhadoras; Sala de amamentação.

\section{ABSTRACT}

Currently the promotion and encouragement of the implementation of breastfeeding support rooms in workplaces is increasing. This theme has been researched, both nationally and internationally, given the importance of providing and supporting the maintenance of breastfeeding, following the recommendations of the World Health Organization. This also represents a great challenge, especially after the return of women to work, and this is the greatest barrier to the maintenance of breastfeeding by working mothers. Scientific objective: To investigate the scientific evidence included in the national and international literature about the importance of breastfeeding rooms already existing in institutions for the maintenance of breastfeeding by working women. Methodology: This study is a narrative review of the literature regarding breastfeeding by female workers, in the workplace and breastfeeding support rooms in these locations. Searches were conducted in LILACS, Medline, Pubmed databases and in tools such as Google Scholar. Results: According to the available literature, instead of mothers leaving the labor market, they leave babies at school and use surrogates for breast milk or stop breastfeeding. Thus,

\footnotetext{
${ }^{1}$ Enfermagem. Mestranda. Universidade Franciscana (UFN).gabiknob78@ gmail.com;

${ }^{2}$ Psicologia. Doutorado. Universidade Franciscana (UFN). cristinakruel@ ufn.edu.br;

${ }^{3}$ Enfermagem. Doutorado. Universidade Federal de Santa Catarina (UFSC). evanguelia.ufsc@ gmail.com

${ }^{4}$ Nutrição. Doutorado. Universidade Franciscana (UFN). francijb@yahoo.com.br

${ }^{5}$ Odontologia. Doutorado. Universidade Franciscana (UFN). dottopp@gmail.com
} 


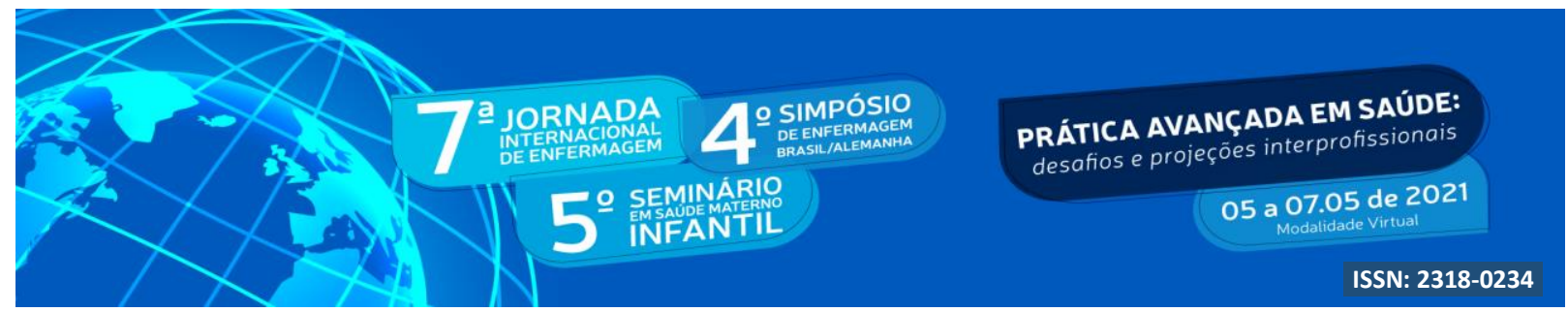

mothers usually recommend access to a room of this type, comfortable and equipped, and to day care centers as support factors, so that they can return to work and continue breastfeeding their children.

Keywords: Breastfeeding; Workplace; Working women; Breastfeeding rooms.

\section{INTRODUÇÃO}

O leite materno (LM) garante as quantidades necessárias de água, carboidratos, lipídeos e proteínas para o desenvolvimento adequado dos lactentes. Ainda, a amamentação possui, além da nutrição, um sentido afetivo, pois é uma fonte de troca de calor, amor e aconchego importantes para o desenvolvimento psíquico e emocional da criança (NUNES, 2015). Crianças amamentadas por um período mais longo possuem vantagens como menor morbidade e mortalidade, maior inteligência do que aquelas que são amamentadas por períodos mais curtos ou não são amamentadas. Como benefícios maternos, a amamentação pode prevenir o câncer de mama, aumentar o intervalo interpartal e reduzir o risco de a mulher desenvolver diabetes ou câncer de ovário (VICTORA et al., 2016).

Segundo orientações da Organização Mundial da Saúde (OMS) e Organização Pan Americana de Saúde (OPAS) na primeira hora de vida, a amamentação deve ser iniciada ainda na sala de parto, e ser mantida como aleitamento materno exclusivo (AME) sem adição de outros alimentos, tanto sólidos, semissólidos ou líquidos até o sexto mês de vida. A partir deste período, deve-se introduzir uma alimentação adequada, mantendo o AME até 2 anos ou mais. Entretanto, o retorno à jornada de trabalho dificulta o cumprimento dessa recomendação, principalmente pelo fato de que a maior parte das empresas brasileiras tem a licença maternidade de quatro meses.

Para amparar o direito à amamentação, o artigo 396 da Consolidação das Leis de Trabalho (CLT), estabelece que, durante a jornada de trabalho, a mãe trabalhadora tem direito a dois descansos especiais, de meia hora cada um, computados na duração do trabalho, para amamentar o próprio filho, até que ele complete seis meses ou, se for possível, sair uma hora mais cedo de seu trabalho, para amamentar. Além disso, se não há possibilidade de amamentar o filho no horário de trabalho, esta mãe terá a necessidade de esvaziar a mama e, para tanto, é essencial uma sala de apoio à amamentação apropriada para a extração e armazenamento de leite. Porém, a realidade é que muitas empresas não respeitam os direitos das mães trabalhadoras que amamentam, com isso surgem as angústias tanto da separação e da 


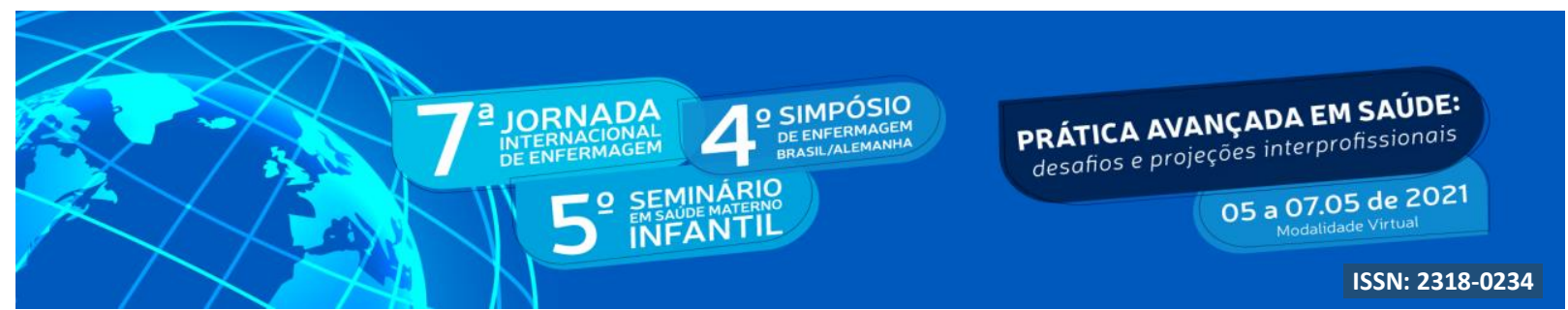

amamentação exclusiva, quanto do medo em relação à produção do leite e o fato de não conseguirem a quantidade de leite suficiente para alimentar o bebê em seu período de trabalho.

\section{OBJETIVO}

Investigar as evidências científicas contempladas na literatura nacional e internacional acerca da importância das salas de amamentação já existentes nas instituições para a manutenção do aleitamento materno por mulheres trabalhadoras.

\section{METODOLOGIA}

Este estudo é uma revisão narrativa da literatura referente ao aleitamento materno por mulheres trabalhadoras, no local de trabalho e às salas de apoio à amamentação nesses locais. Foram realizadas buscas nas bases de dados LILACS, Medline, Pubmed e em ferramentas como o Google Acadêmico. Como critérios de inclusão foram utilizados os descritores: "aleitamento materno", "mulheres trabalhadoras", "local de trabalho" e "salas de amamentação", nas bases de dados que permitem palavras em português e "breastfeeding", "working women", "workplace" e "breastfeeding rooms", nas que permitem pesquisas em inglês.

\section{RESULTADOS E DISCUSSÃO}

Primeiramente, a CF, no artigo $7^{\circ}$, inciso XVIII, busca incentivar a amamentação por meio da garantia à gestante licença maternidade, sem prejuízo do seu emprego e salário, com duração de 120 dias. O mesmo prazo é determinado pela CLT, artigos 392 e 392-A, valendo tanto para a mãe gestante como para a mãe adotante (que adotar ou obtiver guarda judicial de criança). Além disso, o artigo 192, $\S 2^{\circ}$, trata que os períodos de repouso, antes e depois do parto, poderão ser aumentados de 2 semanas cada um, mediante atestado médico (BRASIL, 1943).

Ainda, fim de garantir a exclusividade do aleitamento, a prorrogação da licença maternidade para o prazo de 180 é abordada pela Lei Federal 11.770/2008 (BRASIL, 2008). Essa lei, instituiu o Programa Empresa Cidadã, objetivando a prorrogação da licençamaternidade, sendo ela regulamentada pelo Decreto $\mathrm{n}^{\mathbf{0}}$ 7.052/2009 (BRASIL, 2009). Essa lei considera a prorrogação da licença maternidade, independente do setor, se privado ou se público. A licença maternidade para as servidoras públicas federais foi regulada através do 


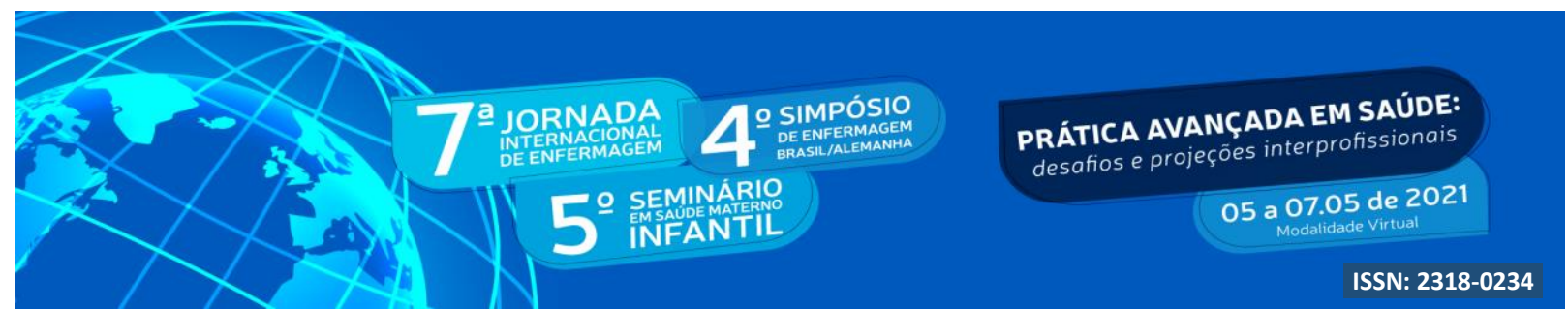

Decreto 6.690/08, na qual é concedida a prorrogação da licença maternidade pelo período máximo de 180 dias, sendo que para as mães adotantes a prorrogação da licença varia de acordo com a idade da criança. No Rio Grande do Sul, a licença maternidade de 180 dias é assegurada à servidora gestante, sem prejuízo da remuneração (RIO GRANDE DO SUL, 2009).

Passado esse tempo da licença maternidade, tem ínicio o desafio de conciliar o trabalho com a amamentação. Nesse sentido, foram localizadas, ao todo, 27 sobre a temática do aleitamento materno de mulheres trabalhadoras no local de trabalho e salas de apoio à amamentação nesses locais, sendo 1 do LILACS e 09 da Medline, 17 no Google Acadêmico, das quais 13 atenderam aos critérios de inclusão. Considerando o idioma, 7 artigos encontrados estavam em inglês e 6 em português. Os estudos foram pesquisados no período de 2013 a 2020 .

A partir desses estudos foi possível perceber que as mulheres que amamentam e que se afastam de seus filhos por causa do trabalho precisam esvaziar suas mamas durante a jornada de trabalho (BRASIL, 2015a). Com isso, as salas de apoio à amamentação são locais para a manutenção da produção do leite, o alívio das mamas ingurgitadas e o armazenamento correto do leite materno. Assim, as salas de amamentação são fundamentais para assegurar a continuidade da amamentação, evitando o desmame (FERNANDES et al., 2017).

Considerando a implantação de salas de amamentação nos locais de trabalho, o estudo realizado por Fernandes et. al. (2017) aponta como principais dificuldades o espaço físico e a questão dos custos, sendo que dentre as 20 empresas pesquisadas, todas não possuíam sala de apoio à amamentação, mas quase metade dessas (45\%) disponibilizavam algum espaço caso a trabalhadora que amamenta necessitasse. Já sobre a questão de custos, Nardi et al. (2020) indicam, ao contrário do apontado por Fernandes et. al. (2017), que a implantação e manutenção de salas de amamentação representam baixo custo, mostrando que não há um consenso sobre esse ponto.

Essa situação apresentada anteriormente, na qual as empresas não destinam um lugar apropriado para a trabalhadora que amamenta esvaziar as mamas durante a jornada de trabalho é uma realidadde brasileira (FERNANDES et al., 2017). Isso porque, em 2019, o Ministério da Saúde apontou que existiam 228 salas de apoio à amamentação espalhadas pelo país, sendo 6 encontradas no Rio Grande do Sul. Com isso, é perceptível a que há a necessidade de um grande avanço nesse sentido. 


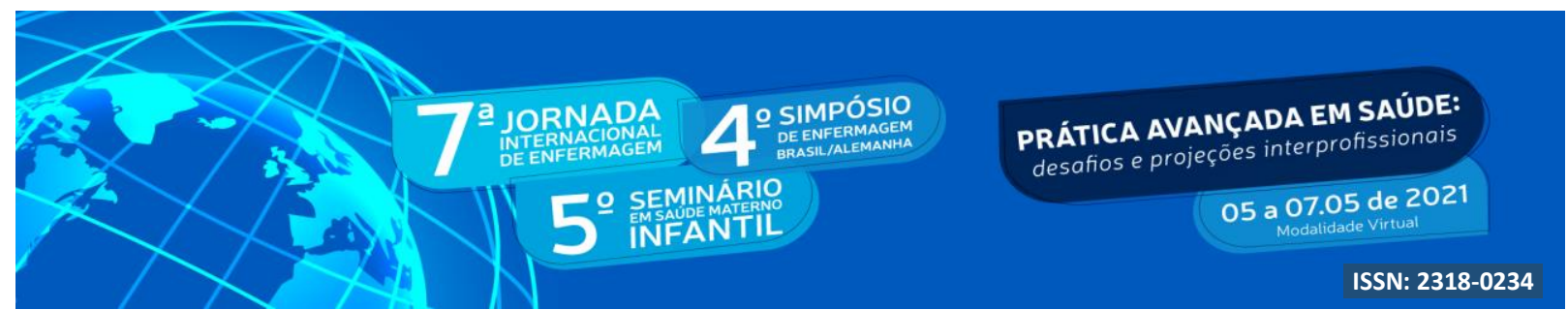

A implantação das sala é importante pois, conforme citado por Kim et al., em 2019, foram relatados significativos aumentos no início da amamentação, na exclusividade ou na duração da amamentação entre as mães que estavam em locais de trabalho com programas voltados a lactação comparado àqueles que não estavam. Além disso, as empresas que tem esse local à disposição das trabalhadoras apresentam absenteísmo reduzido, produtividade aprimorada, maior satisfação e lealdade do empregado, menor perda de pessoal qualificado devido à maternidade e uma imagem corporativa mais positiva (LEON-LARIOS et al., 2019; URIBE et al., 2019).

Assim, são importantes ações de incetivos a implantação e utilização das salas de amamentação. Como ações de incentivo, podemos citar a atuação dos gerentes de recursos humanos. Estes podem realizar avaliações objetivas relacionadas com as necessidades das funcionárias acerca da amamentação e destacar como o apoio à amamentação beneficia todos (FERNANDES et al., 2018; URIBE et al., 2019).

Outro incentivo a manutenção da amamentação, também envolvendo as salas de amamentação, é a adoção de intervalos para a extração do leite materno, uma vez que as mulheres com acesso a intervalos e salas de lactação no trabalho tem 2,3 vezes mais probabilidade de amamentar exclusivamente se comparadas às mulheres que não possuem essas acomodações (KIM; SHIN; DONOVAN, 2019).

O estudo de Tsai (2014a), realizado em Taiwan, revela que muitas vezes as empresas, oferecem intervalos às mulheres que amamentam, mas essas não os usam efetivamente. A partir disso, o estudo mostra a importância de salas de amamentação limpas e incentivo dos colegas, para aumentar a intenção da mãe de utilizar as pausas no trabalho para a extração de leite nas salas de amamentação.

Enfrentados os desafios relativos ao incentivo e a necessidade da amamentação, é necessário avaliar a concreta instalação das salas de amamentação. As salas de amamentação devem ser exclusivas e ser um ambiente confortável e limpo (TSAI, 2013). Diante desta necessidade, as empresas, ao implantar suas salas de amamentação, devem seguir normas técnicas, como a Nota Técnica intitulada "Sala de Apoio à Amamentação em Empresas", que posteriormente foi aprovada como Portaria nº193, em 23 de fevereiro de 2010 (BRASIL, 2010), elaborada pelo o Ministério da Saúde em conjunto com a Agência Nacional de Vigilância Sanitária (ANVISA). Esta portaria tem como objetivo, orientar a instalação de salas de apoio 


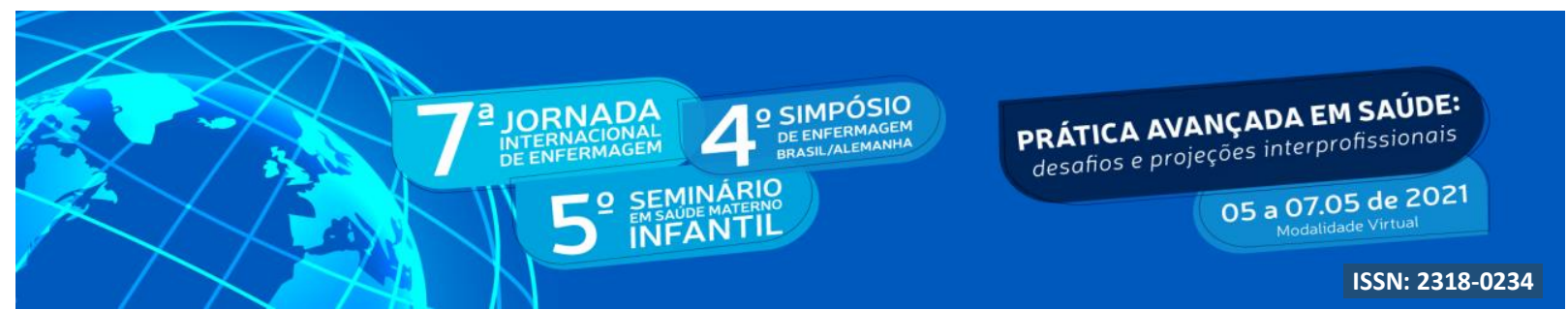

salas de apoio à amamentação em empresas públicas ou privadas e a fiscalização desses ambientes pelas vigilâncias sanitárias locais.

\section{CONCLUSÃO}

O estudo mostrou que a licença maternidade é um direito da mulher e visa incentivar a amamentação nos primeiros meses de vida do recém-nascido, porém o desafio relacionado à amamentação ocorre a partir do momento em que a mulher retorna ao trabalho. Com isso, é evidente a necessidade um amadurecimento por parte das empresas para a implantação de salas de amamentação, e os principais incentivos em relação a instalação e utilização das salas de amamentação são as ações de gerentes, colegas e da própria mãe, além da implantação seguir as normas técnicas para promover um ambiente exclusivo, calmo e limpo.

\section{REFERÊNCIAS}

BRASIL, Casa Civil. Lei 11.770, de 9 de setembro de 2008. Cria o Programa Empresa Cidadã, destinado à prorrogação da licença-maternidade mediante concessão de incentivo fiscal, e altera a Lei no 8.212, de 24 de julho de 1991. Diário Oficial da União de 10 de setembro de 2008.

BRASIL. DECRETO-LEI No 5.452, de 1 de maio de 1943. Aprova a Consolidação das Leis do Trabalho. Diário Oficial da União de 9 de agosto de 1943.

BRASIL, Ministério da Saúde. Portaria n⿳0 193, de 23 de fevereiro de 2010b. Aprova a Nota Técnica Conjunta $n^{\circ}$ 01/2010 Anvisa e Ministério da Saúde, conforme anexo, que tem por objetivo orientar a instalação de salas de apoio à amamentação em empresas públicas ou privadas e a fiscalização desses ambientes pelas vigilâncias sanitárias locais.

BRASIL, Ministério da Saúde. Guia para implantação de salas de apoio à amamentação para a mulher trabalhadora. Ministério da Saúde. Brasília: Ministério da Saúde, 2015.

FERNANDES, V. M. B.; DOS SANTOS, E. K. A.; ERDMANN, A. L.; DE PIRES, D. E. P.; ZAMPIERI, M. F. M.; GREGÓRIO, V. R. P. Implantação de salas de apoio à amamentação em empresas públicas e privadas: potencialidades e dificuldades. Revista gaúcha de enfermagem, v. 37, p. 1-9, 2017.

FERNANDES, V. M. B.; DOS SANTOS, E. K. A.; ZAMPIERI, M. F. M.; GREGÓRIO, V. R. P.; HERNANDES, M. J.; RIBEIRO, L. C. Manager's conduct related to the breastfeeding support at the workplace. Texto e Contexto Enfermagem, v. 27, n. 3, p. 1-12, 2018. 


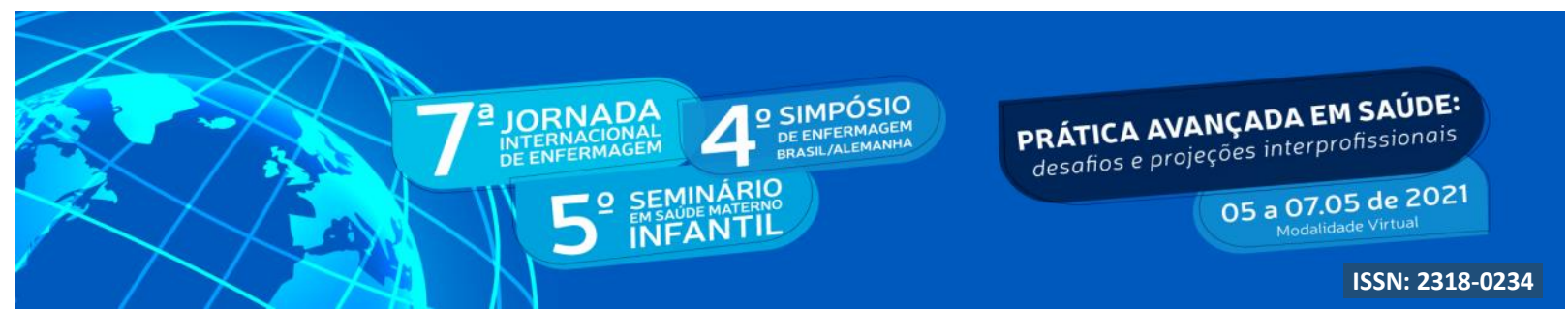

KIM, J. H.; SHIN, J. C.; DONOVAN, S. M. Effectiveness of Workplace Lactation Interventions on Breastfeeding Outcomes in the United States: An Updated Systematic Review. Journal of Human Lactation, [S. l.], v. 35, n. 1, p. 100-113, 2019. DOI: $10.1177 / 0890334418765464$.

LEON-LARIOS, F.; PINERO-PINTO, E.; ARNEDILLO-SANCHEZ, S.; RUIZ-FERRON, C.; CASADO-MEJIA, R.; BENITEZ-LUGO, M. Female employees' perception of breastfeeding-friendly support in a public university in Spain. Public Health Nursing, v. 36, n. 3, p. 370-378, 2019.

NARDI, A. L.; VON FRANKENBERG, A. D.; FRANZOSI, O. S.; SANTO, L. C. E. Impact of institutional aspects on breastfeeding for working women: A systematic review. Ciência e Saúde Coletiva, [S. l.], v. 25, n. 4, p. 1445-1462, 2020. DOI: 10.1590/141381232020254.20382018 .

NUNES, Leandro Meirelles. Importância do aleitamento materno na atualidade. Boletim Científico de Pediatria, v. 4, n. 3, p. 55-58, 2015.

RIO GRANDE DO SUL. Lei no 13.117, de 5 de janeiro de 2009. Altera os arts. 141, 143 e 144, e revoga o art. 142 da Lei Complementar $\mathrm{n}^{\circ}$ 10.098, de 03 de fevereiro de 1994, que dispõe sobre o Estatuto e Regime Jurídico Único dos Servidores Públicos Civis do Estado do Rio Grande do Sul e inclui alterações na Lei Complementar $n^{\circ} 10.990$, de 18 de agosto de 1997, que dispõe sobre o Estatuto dos Servidores Militares da Brigada Militar do Rio Grande do Sul e dá outras providências. Diário Oficial Estadual 003, de 06 de janeiro de 2009.

TSAI, S. Y. Impact of a breastfeeding-friendly workplace on an employed mother's intention to continue breastfeeding after returning to work. Breastfeeding Medicine, v. 8, n. 2, p. 210 $216,2013$.

TSAI, S. Y. Employee perception of breastfeeding-friendly support and benefits of breastfeeding as a predictor of intention to use breast-pumping breaks after returning to work among employed mothers. Breastfeeding Medicine, v. 9, n. 1, p. 16-23, 2014. a.

URIBE, A. L. M.; BOLTON, T. A.; WOELKY, K. R.; OLSON, B. H. Exploring Human Resource Managers' Decision-Making Process for Workplace Breastfeeding-Support Benefits Following the Passage of the Affordable Care Act. Maternal and Child Health Journal, v. 23, n. 10, p. 1348-1359, 2019.

VICTORA, C. G. et al. Breastfeeding in the 21st century: Epidemiology, mechanisms, and lifelong effect. The Lancet, v. 387, n. 10017, p. 475-490, 2016. 